\title{
The ISOLPHARM project: A New ISOL production method of high specific activity beta-emitting radionuclides as radiopharmaceutical precursors
}

\author{
A. Andrighetto, ${ }^{a}$ F. Borgna, ${ }^{b}$ M. Ballan, ${ }^{a}$ S. Corradetti, ${ }^{a}$ E. Vettorato, ${ }^{b}$ A. Monetti, ${ }^{a}$ M. Rossignoli, ${ }^{a}$ \\ M. Manzolaro, ${ }^{\mathrm{a}}$ D. Scarpa, ${ }^{\mathrm{a}}$ G. Prete, ${ }^{\mathrm{a}}$ and N. Realdon ${ }^{\mathrm{b}}$ \\ ${ }^{a} I N F N$, Laboratori Nazionali di Legnaro, Viale dell'Università, 2, 35020 Legnaro (PD), Italy \\ ${ }^{b}$ Department of Pharmaceutical Sciences, University of Padua, Via Marzolo, 5, 35100 Padova, Italy
}

\author{
Published 10 July 2018
}

\begin{abstract}
The ISOLPHARM project explores the feasibility of exploiting an innovative technology to produce extremely high specific activity beta-emitting radionuclides as radiopharmaceutical precursors. This technique is expected to produce radiopharmaceuticals that are virtually mainly impossible to obtain in standard production facilities, at lower cost and with less environmental impact than traditional techniques. The groundbreaking ISOLPHARM method investigated in this project has been granted an international patent (INFN). As a component of the SPES (Selective Production of Exotic Species) project at the Istituto Nazionale di Fisica Nucleare-Laboratori Nazionali di Legnaro (INFN-LNL), a new facility will produce radioactive ion beams of neutronrich nuclei with high purity and a mass range of 80-160 amu. The radioactive isotopes will result from nuclear reactions induced by accelerating $40 \mathrm{MeV}$ protons in a cyclotron to collide on a target of $\mathrm{UC}_{\mathrm{x}}$. The uranium in the target material will be ${ }^{238} \mathrm{U}$, yielding radioactive isotopes that belong to elements with an atomic number between 28 and 57. Isotope separation on line (ISOL) is adopted in the ISOLPHARM project to obtain pure isobaric beams for radiopharmaceutical applications, with no isotopic contaminations in the beam or subsequent trapping substrate. Isobaric contaminations may potentially affect radiochemical and radionuclide purity, but proper methods to separate chemically different elements can be developed.
\end{abstract}

Keywords: Radiopharmaceuticals; cyclotron; beta-emitting.

\section{Introduction}

Radiopharmaceuticals are medicines that deliver a defined dose of radiation to a target tissue for diagnostic or therapeutic procedures (depending on the mechanism of decay). High-penetrating radiation, such as $\gamma$ emission, is mainly used for the early diagnosis of tumors and inflammatory diseases. ${ }^{1}$ Particle emissions capable of inducing cell death, such as $\alpha$ and $\beta^{-}$emissions, are used in cancer therapy and pathologies such as rheumatoid arthritis. The goal of radionuclide therapy is to deliver a cytotoxic level of radiation to a disease site. $^{2}$

Radiopharmaceuticals usually contain two parts: a radioactive core and a carrier system. The carrier allows the precise deposition of radiation onto a malignant cell population, thus avoiding damage to healthy tissues. The physical production of a

This is an Open Access article published by World Scientific Publishing Company. It is distributed under the terms of the Creative Commons Attribution 4.0 (CC-BY) License. Further distribution of this work is permitted, provided the original work is properly cited. 
radioactive core is a point of difficulty, owing to high production costs, low-reaction cross-sections, and issues in product purity under current techniques. This is particularly true in the case of $\beta$-emitting radionuclides.

$\beta$-emitting radionuclides are produced mainly by direct reaction in dedicated targets using neutrons from nuclear reactors. These reactions produce a large number of isotopes and different nuclei in the target. The chemical methods used to extract the desired radionuclides lead to a considerable amount of carrier. The presence of this carrier reduces specific activity, such that the ratio between the activity of the radioisotope (in terms of radioactivity) and the mass of the element taken into account ${ }^{3}$ is very lowwhereas high specific activity is essential for both therapeutic and diagnostic radiopharmaceuticals.

The imperative of high specific activity is especially critical in radioimmunotherapy (RIT) and peptide-receptor radionuclide therapy (PRRT), since cancerous cells have only a few selective sites in which the radiopharmaceutical can exert its action. High specific activity is required to block these sites with tumor-seeking agents that carry the radioactive isotope and not the cold isotope, which has no therapeutic effect. For diagnostic purposes, high specific activity allows the use of a very small amount of radiotracer, which helps maintain the physiological aspect of the process as much as possible. $^{3}$

Using accelerators that employ the isotope separation on line (ISOL) technique may prove effective in producing radionuclides for radiopharmaceuticals, because selecting different beams from a production target (mass selection) enables the production of radiopharmaceuticals with high specific activity - close to theoretical values. ISOL is established as a primary technique for the on-line production of high-intensity and highquality radioactive ion beams. ${ }^{4}$ New construction at Istituto Nazionale di Fisica Nucleare-Laboratori Nazionali di Legnaro (INFN-LNL) will serve the institution's SPES project (Selective Production of Exotic Species) by allowing the production of radioactive-ion beams of neutron-rich nuclei with high purity and a mass range between 80 and 160 amu. $^{5}$ INFN's groundbreaking idea of exploiting ISOL capability for radiopharmaceutical purposes (the ISOLPHARM project) has been granted an international patent.

The steps for radiopharmaceutical preparation are as follows:

(i) Trap the radionuclide of interest in the beam by the construction and placement of a suitable substrate.

(ii) Prepare a medicinal product compatible with the method of administration.

(iii) Guarantee highest quality by compliance with the principles of good manufacturing practice (GMP) for radiopharmaceuticals.

\section{The ISOLPHARM Project at LNL}

ISOLPHARM will begin by performing a feasibility study using the radioactive-ion beams (RIBs) produced in the second-generation Italian ISOL facility (SPES) as a new 
technique for producing extremely pure radionuclides for nuclear medicine. The goal is carrier-free radiopharmaceuticals, achieved by capitalizing on the extreme purity of ISOL radioactive beams. A wide range of experts must contribute to this project, including experts in nuclear physics, engineering, technological production, and radiopharmacy, engaged through a strategic collaboration between the Legnaro National Laboratories of INFN (the facility site) and the department of pharmaceutical and pharmacological sciences at the University of Padua.

At SPES, radioactive isotopes will be obtained through nuclear reactions induced by $40 \mathrm{MeV}$ protons accelerated by a new LNL cyclotron, which will bombard a multifoil target with discs of carbon-dispersed uranium carbide $\left(\mathrm{UC}_{\mathrm{x}}\right)^{6}$ spaced to dissipate the $8-\mathrm{kW}$ heat generated by the reaction. The uranium in the target material will be ${ }^{238} \mathrm{U}$, ensuring that the radioactive isotopes will belong to the group of elements with atomic numbers between 28 and 57 (between nickel and lanthanum in the periodic table). Most of the produced nuclides will be neutron rich, as indicated in the nuclides chart in Fig. 1.

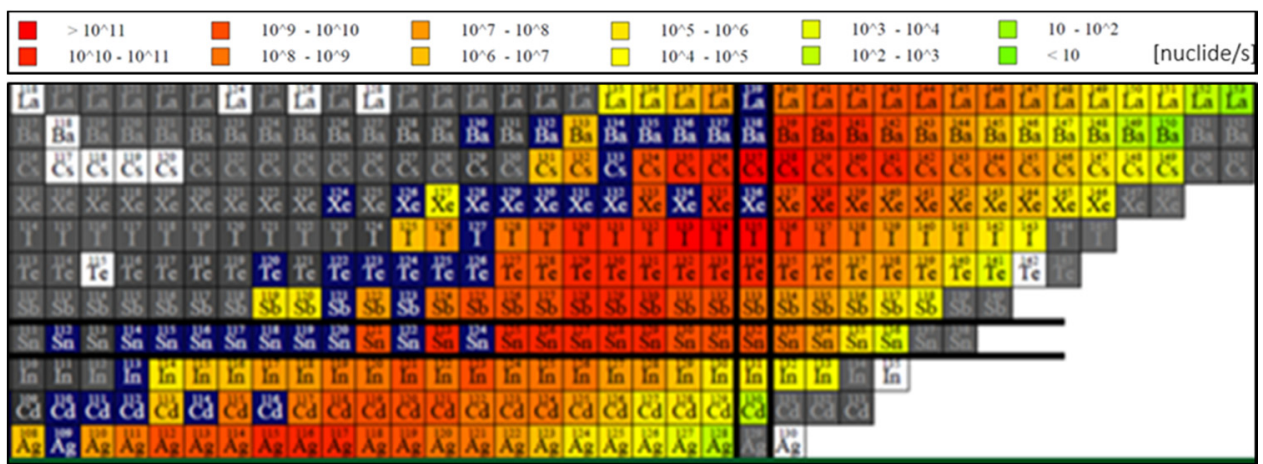

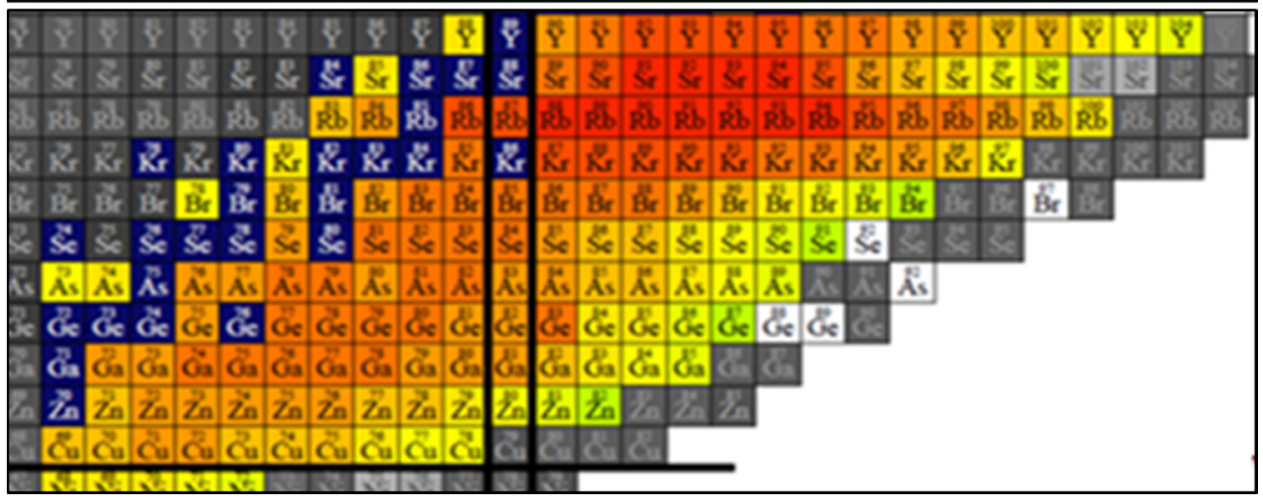

Fig. 1. Chart of nuclides produced from ${ }^{238} \mathrm{U}$ fission target after irradiation with protons at a current of $200 \mu \mathrm{A}$ and energy of $40 \mathrm{MeV}$.

The reaction products will be extracted from the target by evaporation at high temperature (about $2000^{\circ} \mathrm{C}$ ) and forced through a transfer tube towards an ionization 
cavity, where they will be ionized to the $1+$ state. $^{7}$ The ionized isotopes will be accelerated through an electrode at high potential (up to $40 \mathrm{kV}$ ).

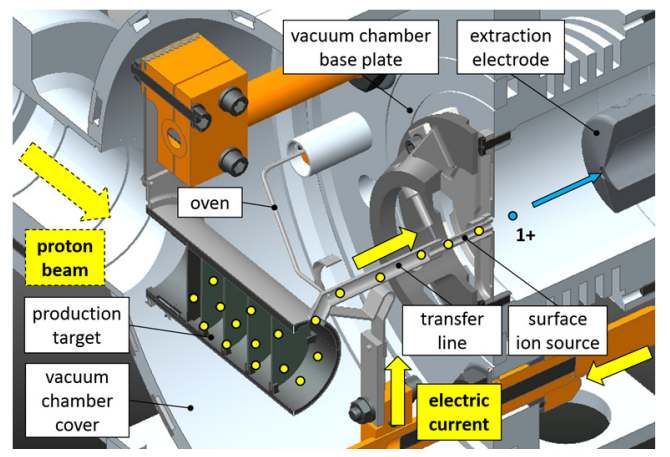

Fig. 2. SPES target layout. The proton beam coming from the left is impinging on the multifoil ${ }^{238} \mathrm{UC}_{\mathrm{x}}$ target.

The formed beam will be directed and focused using various electromagnetic systems, then purified to obtain a pure isotopic beam without contaminants. It will be possible to collect radionuclides of interest using a substrate at the end of the experimental line. Figure 3 illustrates the process.

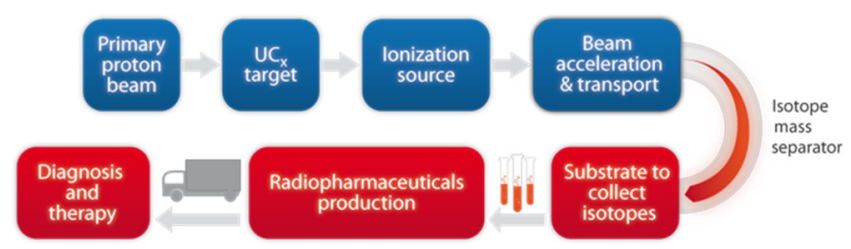

Fig. 3. Steps in radionuclide production and radiopharmaceutical production. Clockwise from top left: the nuclear-physics process, leading to isotope mass separation and, thence, to pharmaceutical applications.

The radioisotope production and collection performed with this technique can produce high-specific-activity materials with a simple procedure, meeting the requirements for application in radiopharmaceuticals. When mass separation is performed effectively $(\Delta M / M$ less than $1 / 200)$, only an isobar chain is present on the collection target, and thus radiopharmaceutical quality is expected to be extremely high. This phenomenon makes the ISOL technique very promising for the production of radionuclides for nuclear medicine.

The uranium-carbide radioisotopes of interest in radiopharmacology are ${ }^{89} \mathrm{Sr},{ }^{8}{ }^{90} \mathrm{Y},{ }^{9}$ ${ }^{125} \mathrm{I},{ }^{10}{ }^{131} \mathrm{I},{ }^{11}$ and ${ }^{133} \mathrm{Xe} .{ }^{12}$ Feasibility studies using stable ion beams for the production of strontium, yttrium and iodine radioisotopes are described in this work.

Initially, ISOLPHARM will produce radionuclides already on the market - the innovation will lie in producing them carrier free. As a second step, ISOLPHARM proposes that the LNL research facility study radionuclides coming from a fissile target 
bombarded with high currents and energy; a broad fission spectrum can be thus obtained, and promising radionuclides spotted.

\subsection{Experimental apparatus}

Preliminary tests were performed at LNL using existing experimental apparatus, including a SPES test-bench front end (FE). The FE was designed and developed for the SPES project (see Fig. 4) and is currently used off line, i.e., for the acceleration of stable ion beams. It will be moved to a dedicated bunker in the final SPES building and linked to the cyclotron line.
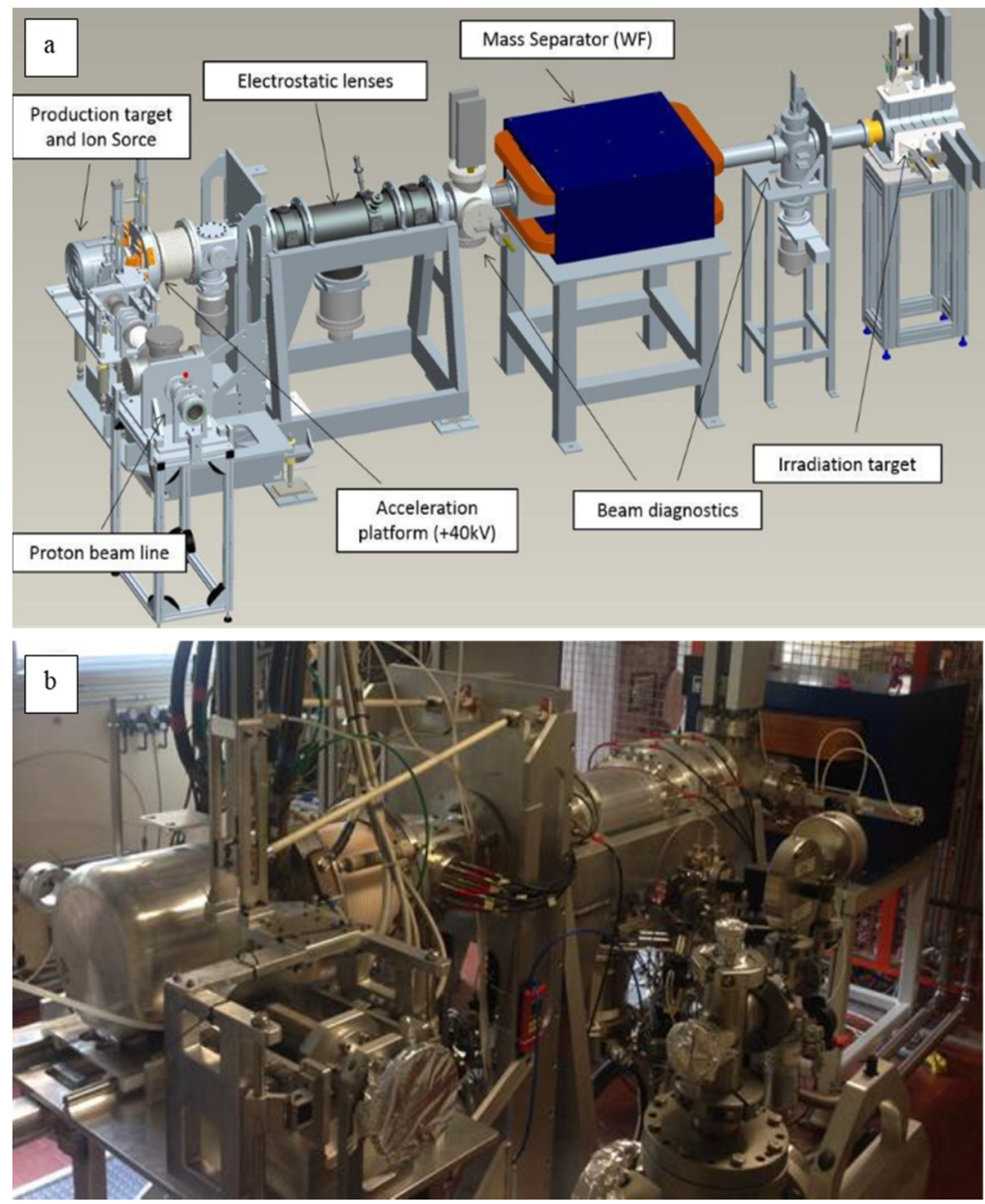

Fig. 4. The SPES FE: (a) a CAD depiction of the SPES FE and main components. (b) The LNL SPES FE, currently off line, is used for experiments with stable ion beams. 
In studying radionuclide production using ISOL to obtain a radiopharmaceutical product, stable isotopes of the same element may be used because they have the same chemical behavior. For this reason, the FE was used to produce stable ion beams and carry out the feasibility tests reported.

The FE contains five functional subsystems: an ion-source complex, a beam-optics subsystem, a Wien filter, and two diagnostic boxes. The ion-source complex is placed in a vacuum chamber that can guarantee the use of the ion source at high temperatures with pressure levels between $10^{-5}$ and $10^{-6}$ mbar. In off-line mode, no uranium-carbide target is installed inside the chamber, but different methodologies are used to introduce the stable isotopes to be ionized and accelerated, depending on the physical state of the element. Gases are introduced through a controlled gas flow and injected in the ionization source via a calibrated leak. Solids are usually in the form of soluble salts, which are dissolved in acidic media and quantitatively deposited onto a tantalum foil, called a mass marker (MM), by evaporation of the solvent. The MM is carefully folded ${ }^{7}$ and inserted into a heated tantalum tube (the "oven") that allows element atomization and injection into the ionization source. The ionization sources used in the experiments described here are of two kinds, according to the first-ionization potential of the element. ${ }^{13,14}$ For elements of the first and second groups, the surface-ion source (SIS) is adopted; for elements with higher electronegativity, the plasma-ion source (PIS) is necessary.

The SPES SIS is a hot-cavity ion source with components mainly made of tantalum (see Fig. 5). ${ }^{7}$ Surface ionization occurs following the interaction of the atoms with a heated surface, causing the loss of an electron and production of a positive singly charged ion. This mechanism works only for those elements with first-ionization potential smaller than $7 \mathrm{eV}$ and a hot cavity made of materials with high work-function values, such as tantalum. ${ }^{15}$ The operating temperature of the source is a crucial parameter in the ionization process.

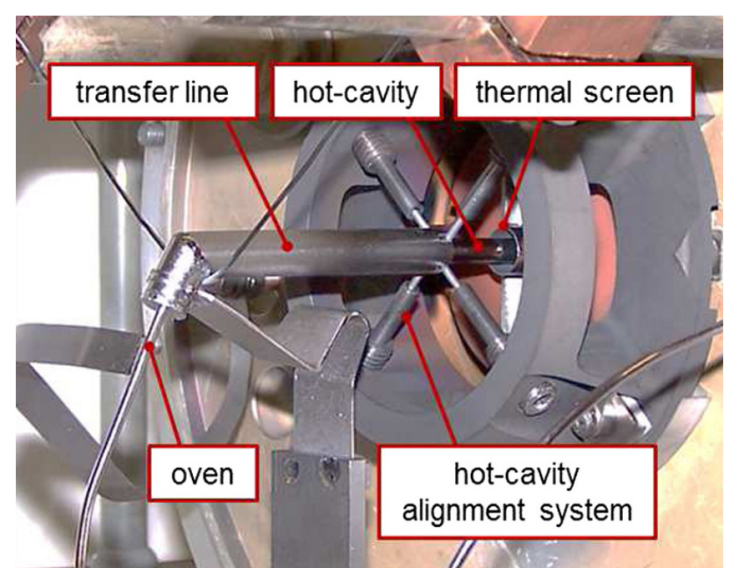

Fig. 5. The SPES SIS. In this off-line test layout, the transfer line is connected to the oven (the tantalum tube used to introduce stable atoms into the source for ionization). The hot-cavity component, the hottest region of the source, is responsible for atomic ionization. With SIS, only low-ionization potential elements can be ionized, in particular those belonging to the first and second group. 
The second kind of source used in tests is the SPES PIS, that is, a forced-electronbeam-induced arc-discharge ion source. ${ }^{13,14}$ As shown in Fig. 6, the PIS is composed mainly of two elements: a tantalum cathode and molybdenum anode. By means of the Joule effect, the cathode is heated and electrons are emitted from its surface by thermionic emission. Electrons then impinge onto the anode, which is kept at $150 \mathrm{~V}$ with respect to the rest of the source; the ionization process is strictly connected to the electron current generated, which depends on the temperature of the cathode. PIS is also suitable for ionizing elements with high first-ionization potential.

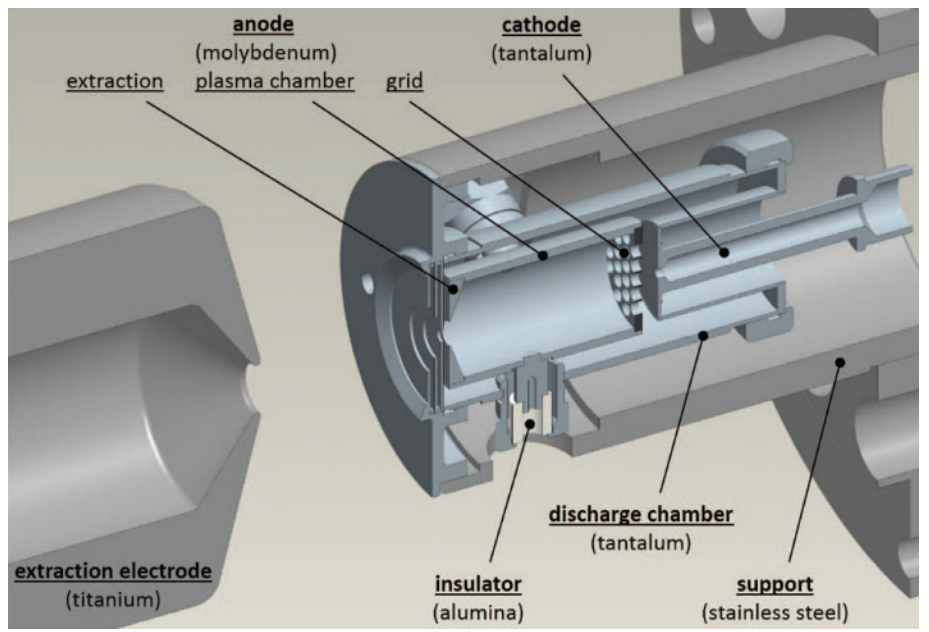

Fig. 6. SPES plasma-ion source (PIS) CAD project. The cathode at right is heated to generate electrons, which are accelerated towards an anode at $150 \mathrm{~V}$. Behind the grid, a plasma of electrons, buffer gas, and 1+ ions is created.

Between the ion source and the extraction electrode, a $25 \mathrm{kV}$ platform is set. The extracting voltage available in this apparatus may range up to $40 \mathrm{keV}$. The beam-optics subsystem comprises a set of electrostatic steerers and a quadrupole triplet that allow, respectively, beam alignment and focusing. In the first diagnostic box is a Faraday cup for beam-intensity monitoring and a grid-based beam-profile detector. The beam passes through a Wien filter, an electromagnetic mass separator used in the FE to generate a pure isobaric beam, thanks to the deviation of undesired masses and a slitted subsystem. A second diagnostic box is installed after the mass separator, consisting of a Faraday cup, a grid-based beam-profile detector, and an emittance meter.

To simulate radionuclide production, a substrate of pharmaceutical grade is positioned at the end of the line, immediately after the emittance meter, to collect the accelerated stable ions desired. 


\subsection{Description of experimental activities}

To collect accelerated ions of interest, different substrates were designed and produced. The technical requirements of a material center around mechanical resistance, resistance to pressures of $10^{-5}-10^{-6}$ mbar, nontoxicity, and the ease and speed of transforming the substrate into a radiopharmaceutical. Whether isobaric contaminants were present was also an important criterion-chemical purification is a crucial step in obtaining radionuclide and chemical purity, so the material must be suitable for isotope recovery and purification.

Among biocompatible materials, sodium chloride was chosen for the deposition of strontium and yttrium. For iodine, activated carbon was the material of choice. In all cases the powders, alone or blended with ligands, were compressed into compact disks with a diameter of $40 \mathrm{~mm}$.

\subsubsection{Sodium chloride}

Sodium chloride was demonstrated ideal for deposition, as completely biocompatible with human administration (oral or injected), having good solubility, and possibly conducing to dissolution in biocompatible media after beam trapping.

Sodium-chloride disks were obtained by pressing different amounts of sieved $(300 \mu \mathrm{m}) \mathrm{NaCl}$ powder in an industrial press. The mold die produced disks of $40 \mathrm{~mm}$ diameter with the application of 30 tons for five minutes; no ligand was used. For the deposition of strontium, the disks were produced by pressing sodium chloride using TraceSELECT (Fluka Analytical) to avoid traces of strontium already present as a contaminant in other types of sodium chloride. For yttrium deposition, nonTraceSELECT sodium chloride (Sigma Aldrich) was used instead, since no yttrium traces are present.

Various quantities of powder were compressed from $1.8 \mathrm{~g}$ to $3.6 \mathrm{~g}$ and tested for mechanical resistance using Stable Micro System's TA-HDi texture analyzer. The measurement was carried out by lowering the probe to $40 \%$ of disk thickness, with the disk fixed on a tailored support on a flange at the end of the FE.

For future production of ${ }^{89} \mathrm{Sr}$, no isobaric impurities will be present in the beam. In the case of ${ }^{90} \mathrm{Y}$ production, the presence of contaminants cannot be avoided with mass separation because of the long half-life of ${ }^{90} \mathrm{Sr}$. For this reason, the purification process of yttrium from strontium is currently under study at the department of pharmaceutical and pharmacological sciences in Padua University.

\subsubsection{Activated carbon}

For iodine deposition, activated carbon was chosen as the secondary target material. Sodium chloride could not be used because it has, as an intrinsic contaminant, iodine in the anion forms of iodate and iodide. Moreover, iodine is gaseous at operating-pressure conditions $\left(10^{-5}-10^{-6} \mathrm{mbar}\right),{ }^{16}$ potentially allowing iodine ions to be lost immediately 
after implantation in the secondary target. Because it can trap iodine, activated carbon was chosen as ideal for matrix development. ${ }^{17,18}$

To obtain a solid and compact matrix, various ligands were considered. For cellulose derivatives and lipophilic ligands, powders were compressed directly, using different amounts of ligand (15\% or $30 \%$ of microcrystalline cellulose, $3 \%$ stearic acid). ${ }^{19}$ In the case of sugars (sorbitol), activated carbon was pre-granulated with sorbitol at $10 \%$ and $30 \% \mathrm{p} / \mathrm{v}$, heated to dry overnight at $40^{\circ} \mathrm{C}$, then pressed.

For polymers, a convenient hot pressure process had to be established. Polyvinyl alcohol (PVA) proved a good ligand for activated carbon. ${ }^{20}$ Various percentages of PVA were tested, following pre-granulation. Powder was compressed for $10 \mathrm{~min}$ at $180^{\circ} \mathrm{C}$, and two tons of pressure were applied to a mold of $40 \mathrm{~mm}$ diameter.

The obtained activated-carbon-PVA $(50 \% \mathrm{~m} / \mathrm{m})$ disks were characterized for morphology, specific surface area, mechanical resistance, and weight loss in vacuum. To characterize disk-surface morphology, SEM (scanning electron microscopy) was performed using a Tescan Vega $3 \mathrm{XMH}$; to verify porosity, a physisorption analyzer was used (the Micromeritics ASAP 2020). Mechanical resistance and weight loss in vacuum were tested as for sodium-chloride matrixes.

\subsubsection{Analytical methods}

An analytical method was established for each element to quantify the effective quantity of atoms deposited on the substrate after beam irradiation; both the nature of the element and of the matrix were considered.

After irradiation, sodium-chloride disks were dissolved in a proper solvent for chemical quantitative analysis. For strontium, atomic-absorption spectroscopy with atomization was used in a graphite furnace (GF-AAS), specifically the Varian GTA 110 with PSD 120 auto sampler. A hollow Heraeus cathode lamp for strontium was used at a wavelength of $460 \mathrm{~nm}$, and a strong acidic matrix was used $\left(\mathrm{HNO}_{3} 0.3 \mathrm{M}\right)$ to reduce nonspecific absorbance phenomena resulting from the presence of $\mathrm{NaCl}^{21}$

Owing to its low volatility, yttrium cannot be observed with AAS, ${ }^{22}$ so ICP-OES (inductive coupled plasma-optic emission spectroscopy) was chosen instead. To minimize the quantity of sodium, disks of $1.8 \mathrm{~g}$ of $\mathrm{NaCl}$ were used for beam deposition.

The analytical method was assessed to be sensible, accurate, precise, and linear in the concentration range. Standards were prepared at $2 \mathrm{ppb}, 7 \mathrm{ppb}, 10 \mathrm{ppb}$, and $70 \mathrm{ppb}$ using $\mathrm{HNO}_{3} 0.1 \mathrm{M}+\mathrm{NaCl} 18 \%$ to obtain the same matrix of the sample. The irradiated $\mathrm{NaCl}$ disk was dissolved in $10 \mathrm{ml}$ of $\mathrm{HNO}_{3} 0.1 \mathrm{M}$. For each concentration, three measurements were taken and the data fit to obtain a calibration curve. Yttrium has three main characteristic wavelengths; to choose the best one, wavelength precision, accuracy and limit of detection (LD) were taken into account. ${ }^{22}$ As a result, $371.030 \mathrm{~nm}$ was determined to be the best wavelength for yttrium quantification.

For iodine quantification, a process was developed to extract iodine deposited in the carbon matrix. As reported in the literature, ${ }^{23}$ basic solutions from $\mathrm{NaOH}$ can efficiently extract iodine from activated carbons. $10 \mathrm{ml}$ of a $0.5 \mathrm{M}$ solution of $\mathrm{NaOH}$ were mixed 
with the irradiated substrate. This solution was then treated with $1 \mathrm{M}$ sulfuric acid in order to obtain an acidic $\mathrm{pH}$. The solution was then automatically titrated (using an 848 Titrino Plus from Metrohm) with sodium thiosulfate $0.001 \mathrm{M}$, after pretreatments with bromine water, formic acid, and an excess of potassium iodide. Titration proved accurate and sensitive enough for iodine quantification.

\subsubsection{Ion-beam deposition}

Using the apparatus and techniques outlined, strontium, yttrium, and iodine beams were produced and collected on the substrates for analysis. Table 1 lists the main experimental conditions for each element.

Table 1. Main experimental conditions for the ionization and deposition experiments carried out for the three elements under study.

\begin{tabular}{llll}
\hline & Strontium & Yttrium & Iodine \\
\hline Ionization source & Surface ion source & Plasma ion source & Plasma ion source \\
Starting chemical form & Strontium nitrate & Yttrium chloride & Potassium iodide \\
Mass marker placement & Oven & Transfer line & Oven \\
Secondary target & Sodium chloride, $3.6 \mathrm{~g}$ & Sodium chloride, $1.8 \mathrm{~g}$ & Act. carbon 50\% $(\mathrm{w} / \mathrm{w})$ \\
& & & PVA 50\% $(\mathrm{w} / \mathrm{w}), 1.3 \mathrm{~g}$ \\
Analytical technique & GF-AAS $(\lambda=460.70 \mathrm{~nm})$ & ICP-OES $(\lambda=371.03 \mathrm{~nm})$ & Titration \\
\hline
\end{tabular}

Before carrying out the depositions, ionization efficiency and focalization tests were performed. Ionization efficiency tests were conducted by evaporating the material contained in the mass marker and collecting the beam in the second Faraday cup. By integrating the curve of current in time, the number of ionized atoms can be obtained; this quantity can be compared to the number of atoms introduced in the MM to obtain ionization efficiency. To check the accelerated ions qualitatively, several mass scans using the Wien filter were performed. The emittance meter was used to control beam dimensions, position, and divergence for each element and adequately set steerers and quadrupole voltage.

During the deposition experiments with strontium and yttrium, the beam current was periodically checked by introducing the secondary Faraday cup for a few seconds. In the case of iodine, the substrate itself (a conductive material) was used as a third Faraday cup, allowing monitoring of the iodine current throughout the deposition. Table 2 summarizes the quantity of deposited elements and the ionization and deposition efficiencies found in the three different cases.

Table 2. Experimental data of deposition of strontium, yttrium and iodine.

\begin{tabular}{llcc}
\hline Element & Deposited atoms & Ionization efficiency \% & Deposition efficiency \% \\
\hline Strontium & $2.8 \cdot 10^{16}$ & $20 \%$ & $41 \%$ \\
Yttrium & $8.0 \cdot 10^{14}$ & $1 \%$ & $100 \%$ \\
Iodine & $9.4 \cdot 10^{16}$ & $20 \%$ & $23 \%$ \\
\hline
\end{tabular}




\section{Conclusions}

From this study, the possibility of using RIBs to obtain high-purity radionuclides was demonstrated, since in all the cases the deposition of stable ion beams was efficient and the recovery quantitative. The chemical purification process for eliminating isobaric contaminants is now under development. This technique opens the possibility of producing a wide range of radionuclides with extremely high levels of purity, both in terms of specific activity, because of the lack of isotopic contaminants, and radionuclidic and chemical purity, because impurities from the beam and targets are very limited, as compared to traditional methods.

\section{References}

1. F. Azaiez, A. Bracco, J. Dobeš, A. Jokinen, G.E. Körner, A. Maj, A. Murphy, P. Van Duppen (eds.), Nuclear Physics for Medicine, NuPECC (2014), ISBN: 9782368730089.

2. Handbook of Nuclear Chemistry (Springer, DOI 10.1007/978-1-4419-0720-2).

3. M.J. Welch, C.S. Redvanly, Handbook of Radiopharmaceuticals, Radiochemistry and Applications (Wiley \& Sons Ltd, Chichester, 2003).

4. T. Nilsson, Nucl. Instr. Meth. Phys. Res. B 317(B), 194 (2013).

5. A. Monetti et al., Eur. Phys. J. A. 51, 128 (2015).

6. S. Corradetti et al., Eur. Phys. J. A. 49, 56 (2013).

7. M. Manzolaro et al., Nucl. Instr. Meth. Phys. Res. B 317(B), 446 (2013).

8. I. Kuroda, Ann. Nucl. Med. 26, 197 (2012).

9. V. Goffredo, A. Paradiso, G. Ranieri, C.D. Gadaleta, Crit. Rev. Oncol. Hematol. 80, 393 (2011).

10. S.B. Schwarz et al., in Brachytherapy, ed. Kazushi Kishi (InTech, 2012).

11. A. Wyszomirska, Nucl. Med. Rev. 15(2), 120 (2012).

12. J.J. Mathews et al., J. Nucl. Med. 49, 771 (2008).

13. M. Manzolaro et al., Rev. Sci. Instrum. 85, 02B918 (2014).

14. M. Manzolaro et al., Rev. Sci. Instrum. 87, $02 \mathrm{~B} 502$ (2016).

15. J. Al-Khalili and E. Roeckl (eds.), The Euroschool Lectures on Physics with Exotic Beams, Vol. II (Springer, Berlin, 2006).

16. R.E. Honig, RCA Review 23, 567 (1962).

17. D.R. Haefner, T.J. Tranter, INL/EXT-07-12299 (Idaho National Laboratory, 2007).

18. P. Paviet-Hartmann, W. Kerlin, S. Bakhtiar, INL/CON-10-19961 (Idaho National Laboratory, 2010).

19. R.C. Rowe, P.J. Sheskey, W.G. Cook, M.E. Fenton (eds.), Handbook of Pharmaceutical Excipients, $7^{\text {th }}$ edition (Pharmaceutical Press, 2012).

20. D. Lozano-Castelló, D. Cazorla-Amorós, A. Linares-Solano, D.F. Quinn, Carbon 40, 2817 (2002).

21. L.J. Prell, D.L. Styris, Spectrochim. Acta. 46, 45 (1991).

22. V. Di Noto, D. Ni, L. Dalla Via, F. Scomazzon, M. Vidali, Analys. 120, 1669 (1995).

23. T. Kaghazchi, N.A. Kolur, R.H. Sabet, Afinidad 542, 338 (2009). 